\title{
Customers' Opinions on Mobile Telecommunication Services in Malaysia using Sentiment Analysis
}

\author{
Muhammad Radzi Abdul Rahim ${ }^{1}$, Yuzi Mahmud ${ }^{3}$ \\ Faculty of Computer and Mathematical Sciences \\ Universiti Teknologi MARA \\ Selangor, Malaysia
}

\author{
Shuzlina Abdul-Rahman² \\ Research Initiative Group of Intelligent Systems \\ Faculty of Computer and Mathematical Sciences \\ Universiti Teknologi MARA \\ Selangor, Malaysia
}

\begin{abstract}
Mobile telecommunication companies in Malaysia have been widely used in the recent decade. There is intense competition among them to keep and gain new customers by offering various services. The reviews of the services by the customers are commonly shared on social media such as Twitter. Those reviews are essential for mobile telecommunication companies to improve their services and at the same time to keep their customers from churning to another company. Hence, this study focuses on the public sentiment on Twitter towards mobile telecommunication services in Malaysia. Data on Twitter was scraped using three keywords: Celcom, Digi, and Maxis. The keywords used to refer to Malaysia's top three mobile telecommunication companies. The timeline for the tweets was between December 2020 until January 2021 and was based on the promotion sales commonly used by the organisation to boost their sales which is called Year End Sales. Corpus-based approach and Machine Learning model using RapidMiner were used in this study, namely, Support Vector Machine (SVM), Naïve Bayes, and Deep Learning. The corpus determines the sentiment from the tweets, either positive, negative, or neutral. The models' performances were compared in terms of accuracy, and the outcome shows that Deep Learning classifiers have the highest performance compared to other classifiers. The results of this sentiment analysis are visualised for easy understanding.
\end{abstract}

Keywords-Sentiment analysis; predictive analytics; RapidMiner; mobile telecommunications

\section{INTRODUCTION}

Sentiment analysis determines a writer's attitude toward a topic or the overall contextual polarity. The author's attitude could be based on his or her judgment or evaluation, affective state (i.e., the author's emotional state at the time of writing), or the intended emotional communication (that is to say, the emotional effect the author wishes to have on the reader) [1]. In other words, sentiment analysis is the classification of text documents, such as user reviews, newsgroup postings, and blogs, based on the polarity of their opinions.

Twitter, Facebook, and Instagram are examples of online social media that allow users to communicate with people worldwide. People can spread data, opinions, statements, and behaviour via social media [2]. They express their thoughts about items or share personal experiences, and they even can influence politics and businesses [3]. For example, practically every large corporation has a Twitter account to keep track of client comments on their services or products. Twitter is a powerful microblogging platform that allows users to post status updates (called "tweets"). These tweets contain a lot of human expressions, such as likes, dislikes, and contributions to many issues [3].

Malaysia's mobile telecommunications services sector has exploded in the recent decade. The competition among existing and new telecommunication service providers has intensified as they aim to keep and gain new consumers by offering a variety of eye-catching promotions and seasonal events. Customers frequently compare the promotions on Twitter. Opinions expressed on Twitter are frequently more influential than other social media because they are made public for all to see and compare. Hence, the motivation of this paper is to develop a model that could predict which telecommunications companies in Malaysia provide the top mobile services for the users.

The following are the contributions of this paper:

- This research proposes a Sentiment Analysis on customers' opinions for mobile telecommunication services in Malaysia.

- A corpus-based approach and several state-of-the-art machine learning models are compared to get the best model.

- The findings are visualised using Microsoft Power BI to understand the results better.

The remaining of this paper is structured as follows: Section II discusses the related works on mobile communications and sentiment analysis, while Section III describes the study's methodology. Section IV highlights the results, and Section V concludes the paper with future works.

\section{LITERATURE REVIEW}

\section{A. Mobile Telecommunication Services in Malaysia}

Mobile telecommunications are the process of sending, transmitting, and receiving information over a distance to communicate [4]. This type of signal transmission is carried out using a mobile device, such as a cell phone, computer, or other wired or wireless devices. Mobile telecommunications have a generation of network standards which are 1G, 2G, 3G, $4 \mathrm{G}$, and 5G. 1G and 2G only give a means of speaking and texting over mobile phones. $3 \mathrm{G}$ allows mobile phone users to 
connect to the internet and send or receive any multimedia transmissions. 4G increases the bandwidth significantly while $5 \mathrm{G}$ is still under development to improve 5G [5].

A study by Dagli and Jenkins [5] stated that mobile phone services are one of the most prominent growing areas in the telecommunication industry while currently holding more than 1.7 billion customers worldwide and targeting around $80 \%$ of the world population as its potential customers. Five (5) big companies dominate the telecommunication industry: Maxis, Celcom, Digi, U Mobile, and Unifi. However, there are three major mobile service providers in Malaysia, namely Maxis, Celcom, and Digi [6]. Smaller carriers with limited coverage in Malaysia and mobile virtual network operators are the rest (MVNOs). Each of these significant corporations has its own set of colours that signify its respective brands. Maxis represents green (post-paid), red (prepaid), blue represents Celcom, and yellow represents Digi.

\section{B. Sentiment Analysis}

Sentiment analysis (SA), also known as opinion mining or contextual mining, is a technique used in Natural Language Processing (NLP), computational linguistics, and text analysis to discover, systematically extract, and quantify subjective data. According to Singh et al. [7], the most common approach is machine learning, a method that needs an essential data set for training and learning the aspects and sentiments associated. Sentiment analysis can be used on any material or object in the form of a customer's voice, such as reviews or responses. For example, if a consumer wants to buy something online, they would usually read reviews on the item or product first, which will help them make the best selection possible [8]. Sentiment analysis allows businesses to understand a user's feelings about a product, service, or brand by converting internet comments into emotion and categorising it as positive, negative, or neutral [9].

Machine learning and lexicon-based approaches are the two most utilised techniques in sentiment analysis [10]. The lexicon-based technique generates flawless dictionaries, whereas the machine learning technique concentrates on feature vectors. The general workflow of sentiment analysis consists of a few processes: goal setting, text preprocessing, parsing the content, text refinement, and analysis and scoring [11].

\section{Machine Learning Algorithms}

1) Support Vector Machine (SVM): Support Vector Machine (SVM) is a supervised machine learning algorithm capable of performing classification, regression, and even outlier detection. The linear SVM classifier works by drawing a straight line between two classes. SVM is a supervised machine learning model for two-group classification issues that uses classification techniques. SVM models can categorise new text after being given labelled training data sets for each category. It is mainly used in text classification, and it comes with a dataset for high-dimensional training [3].

2) Naïve bayes: Naïve Bayes is the supervised machine learning algorithm that uses Bayes theorem for classification problems [11]. The Bayes' Theorem is used to create a collection of classification algorithms known as Naive Bayes classifiers. In Almonajed and Jukić's paper [3], the researchers mentioned that the Naïve Bayes classifier is a popular supervised classifier, furnishes a way to express positive, negative, and neutral feelings in the web text. Naïve Bayes classifier is valuable and efficient for classification purposes [12]. A family of algorithms share a similar idea: each pair of features being classified is independent of the others. It is a numerical-based approach with easy, fast, and high accuracy [13].

3) Deep learning: Deep Learning is a subfield of machine learning concerned with algorithms inspired by the structure and function of the brain called artificial neural networks [6]. Deep learning has emerged as a powerful machine learning technique that learns multiple layers of representations or features of the data and produces state-of-the-art prediction results. Along with the success of deep learning in many application domains, deep learning is also used in sentiment analysis in recent years [6]. Deep Learning is based on a multi-layer feed-forward artificial neural network taught via back-propagation and stochastic gradient descent.

\section{RESEARCH METHODOLOGY}

Machine learning and lexicon-based approaches are the two most utilised techniques in sentiment analysis [11]. The lexicon-based technique generates flawless dictionaries, whereas the machine learning technique concentrates on feature vectors. The general workflow of sentiment analysis consists of a few processes: goal setting, text preprocessing, parsing the content, text refinement, and analysis and scoring [14].

\section{A. Data Collection}

Data was collected by extracting tweets from Twitter using a python tool called Twint. Python is a general-purpose language. It can build anything using Python [15]. Twint is a Python-based Twitter scraping tool that allows scraping tweets from Twitter profiles without using Twitter's API. Twint makes use of Twitter's search operators to scrape tweets from specific people, scrape tweets related to specific themes, hashtags, and trends, and shift out sensitive information from tweets such as e-mail addresses and phone numbers. Twint also uses Twitter to make unique queries that allow it to scrape a Twitter user's followers, tweets they have liked, and whom they follow without requiring any login, API, Selenium, or browser emulation. Tweets scraped were between 1st December 2020 until 31st January 2021. Three keywords are used to scrape the tweets: Celcom, Digi, and Maxis. Any tweets posted on Twitter that contained the keywords and within the durations mentioned above will be automatically scrapped by Twint.

\section{B. Data Preprocessing}

1) Data cleaning: There were seven operators used to clean the datasets. The first operator is called the Retrieve operator. After the datasets had been successfully scraped using Python tools, the datasets were stored in a CSV file. Then the CSV was stored in the RapidMiner repository. The 
Retrieve operator is used to access the stored datasets in the repository and load them into the RapidMiner's process. Then, the second operator is called Filter Examples. The raw datasets collected consist of multiple languages. Since RapidMiner only detects English, only English tweets were chosen. Python tools used in the data collection phase provided a language detector of the tweets. The tweets were labelled by each detected language in the language attribute. Therefore, the Filter Examples operator selected only data labelled as "en", which means English. After only tweets in English were chosen, the following operator used was called the Select Attributes operator. Only four important attributes from 36 common attributes were selected. The selected attributes were time, date, tweet, and username attributes. Then, the operator will keep only the four attributes selected and remove the remaining 32 attributes.

The next operator used was called Replace operator. The value of tweet attributes might contain special characters or punctuation characters. Those characters cannot be processed to perform a Sentiment Analysis. So, those characters need to be removed. Remove Duplicate operator was the fourth operator used in the data preprocessing process. It might be a spamming situation where the customers spam with the same tweets in the real world. Therefore, the operator will remove duplicate data and only keep one. The last operator used in the data preprocessing process was the Filter Examples operator. The operator was used again but with a different purpose. After the datasets go through several processes, the missing values appear. The values were removed due to some factors, including those from a special character or just a punctuation character. Therefore, the operator will remove data with a missing value. Fig. 1 shows a RapidMiner's operator used in the data cleaning process.

2) Data labelling: Fig. 2 shows a RapidMiner's operators used to extract sentiment. The data labelling process is the process to extract the sentiment and classify the texts as either positive, negative, or neutral. The cleaned datasets have been called by using the Retrieve operator. Then, the Extract Sentiment operator was used to extract the tweet's sentiment. A few models can be used to extract the sentiment, and the VADER model has been used in this project. VADER model will produce a compound score for each sentence. The compound score is a metric that calculates the sum of all the lexicon ratings, which have been normalised between - 1 (most extreme negative) and +1 (most extreme positive). Table I shows a compound for each sentiment class.

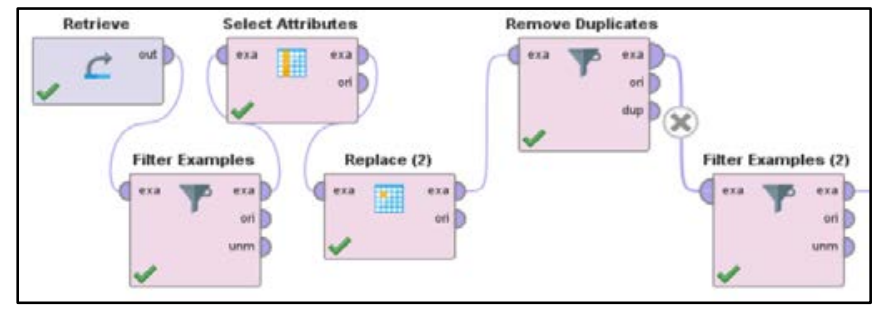

Fig. 1. RapidMiner's Operators used for Data Cleaning.

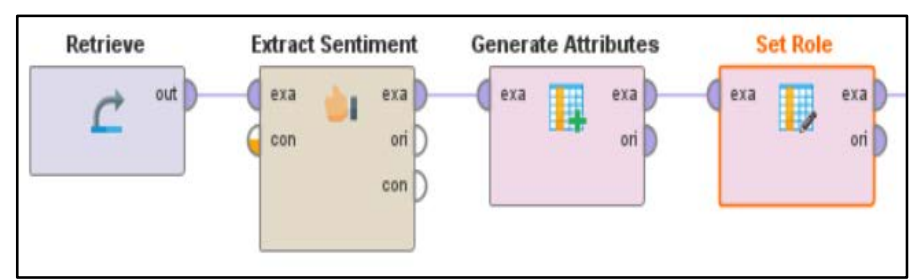

Fig. 2. RapidMiner's Operators use to Extract Sentiments.

TABLE I. COMPOUND SCORE

\begin{tabular}{|l|l|}
\hline Sentiment & Compound Score \\
\hline Positive & $>=0.05$ \\
\hline Neutral & $>-0.05$ and $<0.05$ \\
\hline Negative & $<=-0.05$ \\
\hline
\end{tabular}

After three classes of sentiment have been successfully extracted, the following process sets the role for the sentiment attribute. RapidMiner's operator used was called the Set Role operator. The role of the attribute that has been set will describe how other operators handle the attribute. This operator also will transform the sentiment attribute into a special attribute.

3) Data transformation: Fig. 3 shows the operators applied in the Process Document operator. Only attributes that focused on sentiment analysis were involved in this stage, which is tweet attributes. There are five processes involved in the data transformation stage. The first process was Transform Cases. Transform Cases operator has been applied to transform all characters in the tweet attribute into lower case. The second process was stopped word removal. All stop words, such as frequent terms like a and the, are deleted from multiple word queries to improve search performance. The third process was the tokenisation process, where the Tokenize operator was applied. For this project, non-letter characters have been used as a method for the splitting points. The fourth process was the stemming process, where the Stem operator has been applied. This operator stems from English words using the Porter stemming algorithm, which employs an iterative, rule-based substitution of word suffixes intending to shorten the words to a minimum length. The last data transformation process was generating n-grams by using the Generate n-Grams operator. This operator generates the word n-Grams, which refers to all series of consecutive tokens of length $n$.

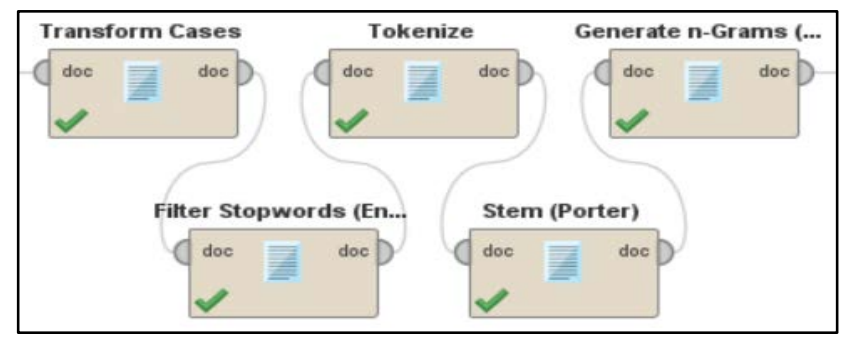

Fig. 3. Operators Applied in Process Document Operator. 


\section{Model Development}

In this project, three machine learning models were compared. The classifiers used were Support Vector Machine (SVM), Naïve Bayes, and Deep Learning. Deep learning is a class of machine learning algorithms that uses multiple layers to progressively extract higher-level features from the raw input [16-17]. Those Machine Learning models will be applied using RapidMiner. After the datasets had been successfully labelled, the result showed an imbalanced class. Datasets are said to suffer the Class Imbalance Problem when highly imbalanced class distributions. The datasets must be equally distributed for each class to get higher accuracy. There are a few methods that can be used to handle imbalanced data. This study used the under-sampling method to obtain an evenly dispersed amount of data to a model. This method investigates the class with the smallest number. Fig. 4 shows RapidMiner's operators used in the modelling process.

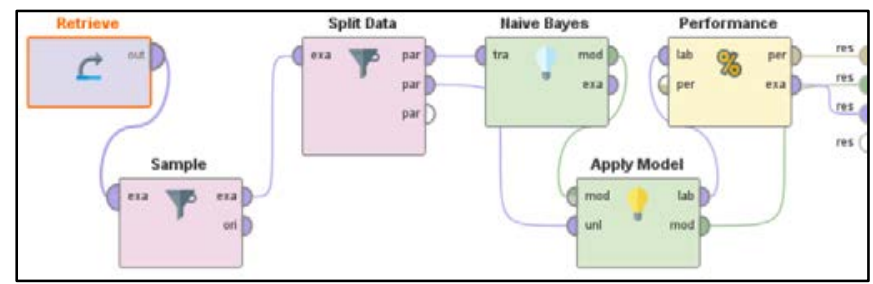

Fig. 4. RapidMiner's Operators used in the Modelling Process.

The first operator used in the modelling process was the Retrieve operator. This operator is used to call the datasets saved in the RapidMiner repository. The second operator used was the Sample operator. This operator was used to solve the imbalanced class problem. The size of the sample has been specified with an absolute sample. Then, the size per class has been set by the smallest number of sizes per class for each dataset. The next operator applied was the Split Data operator. The operator is used to split the datasets into two parts: train data and testing data. The split ratio used in this project was $70: 30,80: 20$, and 90:10 percent. The three ratios were used to find the best ratio which produced the highest accuracy. After the Split Data operator splits the datasets into training and testing data, the training data will be passed to the classifier operator. In contrast, the testing data will be passed to the Apply Model operator. Since this project will compare three different Machine Learning models, three different classifiers will be used.

After the training data has been successfully processed in the classifier operator, the training data also will be passed to the Apply Model operator like the testing data. Therefore, the Apply Model operator will receive both parts of data: training and testing data. Apply Model operators have two types of input received: Model and Unlabeled data. Model input will be received data that model has been trained, while Unlabeled data input will be received testing data. This operator also will apply a model used on the datasets. Not only receive two types of inputs, but this operator will also produce two types of output: Labelled data and model. The input of the Model type was passed without changing to the output through the output of the model type. While the datasets delivered from the output of the labelled type will be passed to the Performance operator. The last operator applied in the modelling process was a Performance operator. This operator is used to evaluate the performance of classification tasks statistically. This operator will return a list of classification task performance criteria values.

\section{Dashboard Development}

The goal for developing the dashboard in this project was to visualise customers' reviews and comparisons between each telecommunication company's customers based on Twitter data. This section goes through the visualisation that may be derived from the sentiment data that has been extracted. The subject under discussion is customers' reviews on Malaysia's top three telecommunication services, and the dashboard was developed using Microsoft Power BI.

\section{RESUlT AND DisCUSSION}

\section{A. Split Ratio Selection}

There are three split ratios used in this project which is 70:30, 80:20, and 90:10 percent ratio. Each split ratio produces a different accuracy, and the split ratio which produces the highest accuracy was selected. Table II until Table IV presents the accuracy result by different split ratios for each dataset. Table II shows the accuracy result for the Celcom dataset. As shown in Tables II to IV, generally, the performance of the three classifiers increases as the sample size increases. In Table II, the Deep Learning classifier appears to be the best model, followed by the Naive Bayes and Support Vector Machines classifier. Similar performance can be seen in Digi and Maxis datasets, in which Deep Learning consistently gave the highest accuracy results.

Based on the accuracies in Table II until Table IV, the 90:10 percent split ratio produced the highest accuracy for each classifier and each dataset. Therefore, the 90:10 split ratio will be used for the following process: finding the best $\mathrm{N}$-gram for the better accuracies produced.

TABLE II. ACCURACY RESUlT FOR CELCOM DATASET

\begin{tabular}{|l|l|l|l|}
\hline Classifier & $\mathbf{7 0 : 3 0}$ & $\mathbf{8 0 : 2 0}$ & $\mathbf{9 0 : 1 0}$ \\
\hline SVM & $49.87 \%$ & $49.94 \%$ & $50.93 \%$ \\
\hline Naive Bayes & $60.84 \%$ & $61.52 \%$ & $61.59 \%$ \\
\hline Deep Learning & $79.17 \%$ & $77.81 \%$ & $81.22 \%$ \\
\hline
\end{tabular}

TABLE III. ACCURACY RESUlt FOR Digi DATASET

\begin{tabular}{|l|l|l|l|}
\hline Classifier & $\mathbf{7 0 : 3 0}$ & $\mathbf{8 0 : 2 0}$ & $\mathbf{9 0 : 1 0}$ \\
\hline SVM & $37.86 \%$ & $38.31 \%$ & $38.72 \%$ \\
\hline Naive Bayes & $52.93 \%$ & $51.31 \%$ & $55.49 \%$ \\
\hline Deep Learning & $65.03 \%$ & $69.73 \%$ & $83.73 \%$ \\
\hline
\end{tabular}

TABLE IV. ACCURACY RESUlt FOR MAXIS DATASET

\begin{tabular}{|l|l|l|l|}
\hline Classifier & $\mathbf{7 0 : 3 0}$ & $\mathbf{8 0 : 2 0}$ & $\mathbf{9 0 : 1 0}$ \\
\hline SVM & $44.84 \%$ & $44.21 \%$ & $45.86 \%$ \\
\hline Naive Bayes & $52.84 \%$ & $52.60 \%$ & $53.19 \%$ \\
\hline Deep Learning & $76.89 \%$ & $76.91 \%$ & $78.25 \%$ \\
\hline
\end{tabular}




\section{B. Model Comparison}

1) Support Vector Machine (SVM): The first classifier tested was the Support Vector Machine. Table V shows the accuracy for the SVM model with three conditions: without nGrams, n-Grams set to 2, and n-Grams set to 4 .

TABLE V. ACCURACY RESUlT FOR SVM MODEL

\begin{tabular}{|c|c|c|c|}
\hline \multirow{2}{*}{ Dataset } & \multicolumn{3}{|l|}{ Accuracy } \\
\hline & Without n-Grams & n-Grams $=2$ & n-Grams $=4$ \\
\hline Celcom & $50.93 \%$ & $52.28 \%$ & $45.18 \%$ \\
\hline Digi & $38.72 \%$ & $49.44 \%$ & $53.98 \%$ \\
\hline Maxis & $45.86 \%$ & $55.40 \%$ & $54.99 \%$ \\
\hline
\end{tabular}

For the conclusion, after the three different datasets have been tested using the SVM classifier, by applying the change of parameter with n-Grams equal to 2 has been set, the accuracy for the classifier for Celcom and Maxis datasets were the highest. While for the Digi dataset, by applying the change of parameter with n-Grams equal to 4 has been set, the accuracy for the classifier was the highest.

2) Naïve bayes: The second classifier that has been tested was Naïve Bayes. Table VI shows the accuracy for the SVM model with three conditions: without n-Grams, n-Grams set to 2, and n-Grams set to 4 .

TABLE VI. ACCURACY RESUlt FOR NAÏVE BAYES MODEL

\begin{tabular}{|c|c|c|c|}
\hline \multirow{2}{*}{ Dataset } & \multicolumn{3}{|l|}{ Accuracy } \\
\hline & Without n-Grams & n-Grams $=2$ & n-Grams $=4$ \\
\hline Celcom & $61.59 \%$ & $62.44 \%$ & $63.62 \%$ \\
\hline Digi & $55.49 \%$ & $56.23 \%$ & $43.49 \%$ \\
\hline Maxis & $53.19 \%$ & $56.58 \%$ & $48.73 \%$ \\
\hline
\end{tabular}

In conclusion, after the three different datasets have been tested using the Naïve Bayes classifier by applying the change of parameter with n-Grams equal to 2 has been set, the accuracy for the classifier for Digi and Maxis datasets will be the highest. While for the Celcom dataset, n-Grams equals 4 produced the highest accuracy.

3) Deep learning: The final classifier that has been tested was Deep Learning. Table VII shows the accuracy for the SVM model with three conditions: without n-Grams, n-Grams set to 2, and n-Grams set to 4 .

TABLE VII. ACCURACY RESUlt FOR DEEP LEARNING MODEL

\begin{tabular}{|l|l|l|l|}
\hline \multirow{2}{*}{ Dataset } & \multicolumn{3}{|l|}{ Accuracy } \\
\cline { 2 - 4 } & Without n-Grams & n-Grams = 2 & n-Grams = 4 \\
\hline Celcom & $81.22 \%$ & $78.00 \%$ & $66.33 \%$ \\
\hline Digi & $83.73 \%$ & $81.22 \%$ & $41.67 \%$ \\
\hline Maxis & $78.25 \%$ & $74.39 \%$ & $59.33 \%$ \\
\hline
\end{tabular}

For the conclusion, after the three different datasets have been tested using Deep Learning classifiers, applying the change of parameter with n-Grams equal to 2 and n-Grams equal to 4 has been set, the accuracy for the classifier will be decreased. While without the n-Grams, the classifiers will perform better. It was different from the SVM and Naïve Bayes classifier.

\section{Dashboard Visualisation}

The dashboard of customers' reviews on Malaysia's top three telecommunication services was developed in 2 pages. Fig. 5 shows the first page of the dashboard. This page was aimed to give a clear view to the readers about the comparison between the top three telecommunication services in Malaysia based on customers' reviews on Twitter. The results are related to customer satisfaction. When the customers feel satisfied, they believe in the brand and become loyal [18]. Based on the visualisation on page 1, it can be concluded that Digi telco has the highest number of positive reviews, while the second-highest was Celcom telco. From that, the readers can decide where the readers might be tended to choose a telco with the highest positive reviews. The readers also were able to read the reviews given by previous users in the table visualisation.

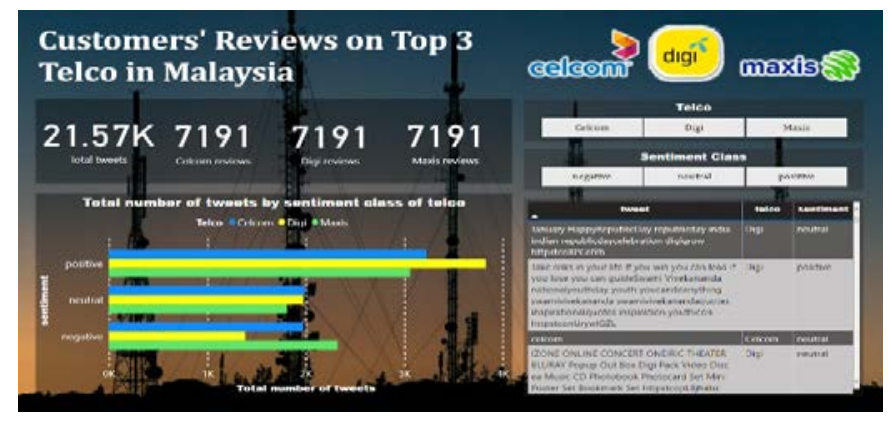

Fig. 5. Page 1 of the Dashboard.

Fig. 6 shows a second page of the dashboard. This page was aimed to provide profound information about the reviews. The readers from the background as a telco's customers might not be interested in this page because most of the telco's customers just want to know the information that can help them decide. At the same time, this page was more focused on telco companies' sites. Based on the line chart on the second page, the telco companies can identify a specific time the reviews were given in the duration selected. From that information, the telco companies might gain new knowledge about the customer's behaviour or what has been suffered by the customers in that time so that the telco companies can decide to maintain customers satisfaction.

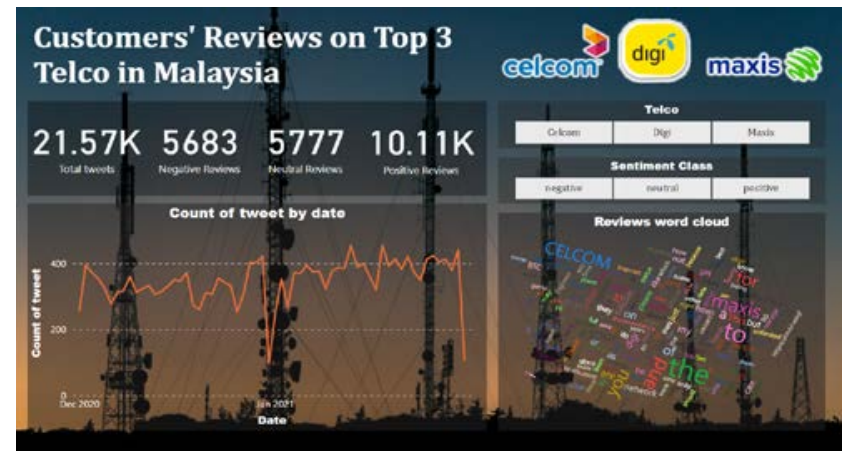

Fig. 6. Page 2 of the Dashboard. 


\section{CONCLUSION}

This study has demonstrated SVM, Naive Bayes, and Deep Learning using a corpus-based approach and machine learning algorithms. Our results showed that the Deep Learning model without n-Grams provides the highest accuracy. The highest value produced for the Celcom dataset is $81.22 \%$ which is $17.6 \%$ better than the Naïve Bayes model and $28.94 \%$ better than the SVM model. Meanwhile, for the Digi dataset, the Deep Learning model produced 83.73\% accuracy, 27.5\% better than the Naïve Bayes model and $29.75 \%$ better than the SVM model. Lastly, for the Maxis dataset, the Deep Learning model produced $78.25 \%$ accuracy, which is $21.68 \%$ better than the Naïve Bayes model and 22.85\% better than the SVM model. The future researcher may consider using a more extensive dataset for better generalisation. This work would reach a wider audience with a mobile application since smartphones are widely used nowadays. Further future work could also include the Malay language during sentiment extraction since Malay is the highest population in Malaysia.

\section{ACKNOWLEDGMENT}

The authors would like to thank the Faculty of Computer and Mathematical Sciences, Universiti Teknologi MARA, Malaysia, for the support throughout this research.

\section{REFERENCES}

[1] N. Sakinah Shaeeali, A. Mohamed, and S. Mutalib, "Customer reviews analytics on food delivery services in Social Media: A Review," IAES International Journal of Artificial Intelligence (IJ-AI), vol. 9, no. 4, p. 691, 2020.

[2] M. Bakri C. Haron, S. Z. Z. Abidin, N. Azmina M. Zamani "Visualisation of crime news sentiment in Facebook," International Journal of Engineering \&amp; Technology, vol. 7, no. 4.38, p. 955, 2018.

[3] Almonajed, O. and Jukić, S., 2021. "Sentiment Analysis on Twitter Data using Big Data." Journal of Engineering and Natural Sciences, 3(1).J. Clerk Maxwell, A Treatise on Electricity and Magnetism, 3rd ed., vol. 2. Oxford: Clarendon, 1892, pp.68-7319.[4] KDnuggets. 2021. "5 Things You Need to Know about Sentiment Analysis and Classification" KDnuggets. [online] Available at: <https://www.kdnuggets.com/2018/ 03/5-things-sentiment-analysis-classification.html> [Accessed 27 August 2021].K. Elissa, "Title of paper if known,” unpublished.

[4] Dagli, O. and Jenkins, G., 2016. "Consumer preferences for improvements in mobile telecommunication services." Telematics and Informatics, 33(1), pp.205-216. Y. Yorozu, M. Hirano, K. Oka, and Y. Tagawa, "Electron spectroscopy studies on magneto-optical media and plastic substrate interface,” IEEE Transl. J. Magn. Japan, vol. 2, pp. 740-741, August 1987 [Digests 9th Annual Conf. Magnetics Japan, p. 301, 1982].

[5] Marcopolis.net. 2021. "Top Telecoms in Malaysia | Malaysia's Largest Telecoms." [online] Available at: <https://marcopolis.net/top-telecomsin-malaysia-malaysia-s-largest-telecoms.htm> [Accessed 27 August 2021].

[6] J. Singh, G. Singh, and R. Singh, "Optimisation of sentiment analysis using machine learning classifiers," Human-centric Computing and Information Sciences, 11-Dec-2017. [Online]. Available: https://hcisjournal.springeropen.com/articles/10.1186/s13673-017-0116-3. [Accessed: 27-Aug-2021].

[7] Etter, M., Colleoni, E., Illia, L., Meggiorin, K. and D’Eugenio, A., 2016. "Measuring Organisational Legitimacy in Social Media: Assessing Citizens' Judgments With Sentiment Analysis." Business \& Society, 57(1), pp.60-97.

[8] Medium. 2021. "A Beginner’s Guide to Sentiment Analysis." [online] Available at: <https://medium.com/@mattkiser/a-beginner-s-guide-tosentiment-analysis-888390a8085a> [Accessed 27 August 2021].

[9] A., V. and Sonawane, S., 2016. "Sentiment Analysis of Twitter Data: A Survey of Techniques." International Journal of Computer Applications, 139(11), pp.5-15.

[10] Godsay, M., 2015. "The Process of Sentiment Analysis: A Study. International Journal of Computer Applications," 126(7), pp.26-30.

[11] N. T. Hazmiza, "Classifying violent elements in role-playing games based on user review using naïve Bayes technique," International Journal of Advanced Trends in Computer Science and Engineering, vol. 9, no. 1.3, pp. 402-407, 2020.

[12] N. Seman and N. Atiqah Razmi, "Machine learning-based technique for big data sentiments extraction," IAES International Journal of Artificial Intelligence (IJ-AI), vol. 9, no. 3, p. 473, 2020.

[13] Singh, S., 2020. "Twitter Sentiments Analysis Using Machine Learning. International Journal of Scientific Research in Computer Science," Engineering and Information Technology, pp.312-320.

[14] N. S. Mohd Shafiee and S. Mutalib, "Prediction of mental health problems among higher education student using machine learning," International Journal of Education and Management Engineering, vol. 10, no. 6, pp. 1-9, 2020.

[15] J. Brownlee, "What is deep learning?,” Machine Learning Mastery, 14Aug-2020. [Online]. Available: https://machinelearningmastery.com/ what-is-deep-learning/. [Accessed: 27-Aug-2021].

[16] Meltwater, E., 2021. "Deep Learning Models for Sentiment Analysis." [online] Underthehood.meltwater.com. Available at: <https://underthehood.meltwater.com/blog/2019/08/22/deep-learningmodels-for-sentiment-analysis/> [Accessed 17 October 2021].

[17] Muhammad, I., Farid Shamsudin, M. and Hadi, N., 2016."How Important Is Customer Satisfaction? Quantitative Evidence from Mobile Telecommunication Market." International Journal of Business and Management, 11(6), p.57. 